\title{
Formation of Interface Bound States on a Graphene-Superconductor Junction in the Presence of Charge Inhomogeneities
}

\author{
Pablo Burset ${ }^{*}$, William Javier Herrera ${ }^{2}$, Alfredo Levy Yeyati ${ }^{1}$ \\ ${ }^{1}$ Departamento de Física Teórica de la Materia Condensada and Instituto Nicolás Cabrera, \\ Universidad Autónoma de Madrid, Madrid, Spain \\ ${ }^{2}$ Departamento de Física, Universidad Nacional de Colombia, Bogotá, Colombia \\ Email: " pablo.burset@uam.es
}

Received November 12, 2012; revised December 17, 2012; accepted January 14, 2013

\begin{abstract}
Interface bound states have been theoretically predicted to appear at isolated graphene-superconductor junctions. These states are formed at the interface due to the interplay between virtual Andreev and normal reflections and provide long-range superconducting correlations on the graphene layer. We describe in detail the formation of these states from combining the Dirac equation with the Bogoliubov-de Gennes equations of superconductivity. On the other hand, fluctuations of the low-energy charge density in graphene have been confirmed as the dominating type of disorder. For analyzing the effect of disorder on these states we use a microscopic tight-binding model. We show how the formation of these states is robust against the presence of disorder in the form of electron charge inhomogeneities in the graphene layer. We numerically compute the effect of disorder on the interface bound states and on the local density of states of graphene.
\end{abstract}

Keywords: Graphene; Superconducting Hybrid Structures; Andreev Reflection

\section{Introduction}

The peculiar electronic band structure of graphene has been the focus of an intense research activity [1]. In graphene, the electronic low-energy properties are governed by a massless relativistic Dirac Hamiltonian which makes the carriers moving in it develop very interesting properties like an electronic spectrum linear with the wave vector and electronic states which are chiral with respect to the pseudospin defined by the two atoms of the crystal unit cell. As a result, graphene exhibits exotic effects like the Klein paradox - perfect transmission through potential barriers [2].

Of particular interest is the case of a graphene layer in contact with a superconducting electrode, where the interplay between superconductivity and the relativistic behavior of charge carriers in graphene can be tested [3]. Graphene is not intrinsically a superconductor but it can easily inherit the bulk properties of other materials when in contact with them. Good contacts can be achieved between lithographically defined superconducting electrodes and graphene layers [4-7]. In such hybrid devices, a superconducting gap is induced by proximity effect on the graphene region underneath the metallic electrodes;

${ }^{*}$ Corresponding author. in these conditions, Andreev processes featuring conversion of electrons into holes in the normal region and the creation of a Cooper pair in the superconductor take place [8]. In a recent experiment using planar $\mathrm{Pb}$ probes a high-quality tunneling spectroscopy has been performed when the $\mathrm{Pb}$ was in the superconducting regime [9].

Several theoretical studies of the electronic and transport properties of graphene-superconductor hybrid structures have been reported in the recent years. The differential conductance and the spectral properties of graphene-superconductor junctions and graphene-based Josephson junctions have been studied within a tightbinding framework and solving the Dirac-Bogoliubov-de Gennes (DBdG) equations under the approximation of neglecting inter-valley scattering [10-19]. The influence of electron-hole inhomogeneities on the Andreev reflection on graphene-superconductor hybrid structures was studied in [20]. The properties of bound states arising from multiple Andreev reflections in a graphene Josephson junction have been analyzed in $[21,22]$. However, the special electronic properties of graphene are such that bound states can be formed even at isolated single junctions. The existence of interface bound states (IBS) was demonstrated in [23] for different edge orientations and doping conditions of the graphene layer. In this work we 
study how robust these bound states are to the effect of disorder on the graphene sample.

This article is organized as follows: First, we discuss in Section 2 the formation of interface bound states at a graphene-superconductor interface by matching of the solutions of the DBdG equations. In Section 3, we introduce the microscopic tight-binding model which includes a superlattice potential that is used to simulate the effects of disorder. Finally, in Section 4 we present our main results and end with some conclusions.

\section{Interface Bound States}

The special electronic properties of graphene are such that bound states can be formed at isolated graphenesuperconductor junctions [23]. The mechanism for the emergence of these states can be understood from the scheme depicted in the left panel of Figure 1. As is usually assumed the junction can be modeled as an abrupt

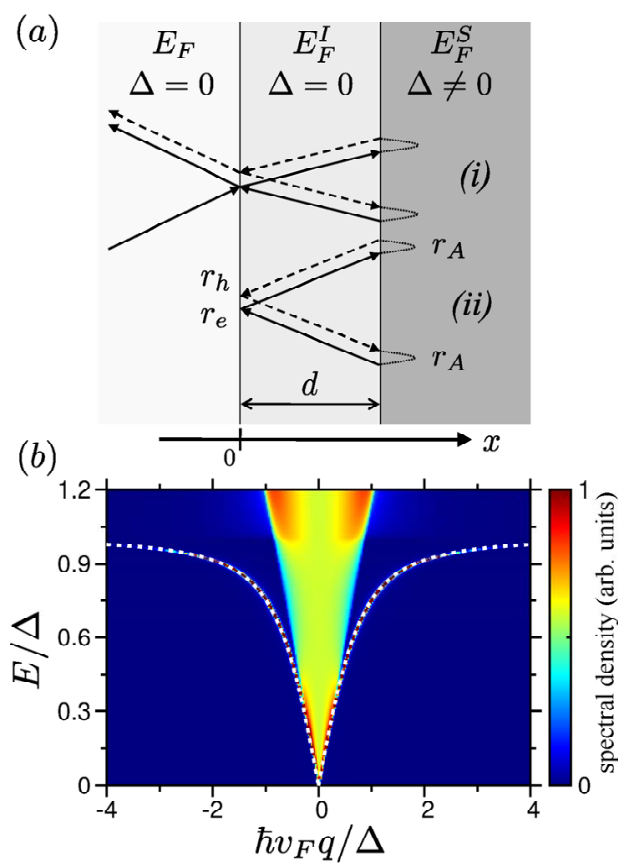

Figure 1. (a) Simple model for the emergence of IBSs illustrating the scattering processes taking place at a graphene-superconductor interface with an intermediate heavily doped normal graphene region of width $d$. Cases (i) and (ii) correspond to the situation with $\hbar v_{F}|q|<\left|E_{F}-E\right|$ and $\hbar v_{F}|q|>\left|E_{F}-E\right|$, respectively, where $D>E>E_{F}$. (b) Map of the spectral density of states where the light regions indicate a higher spectral density showing the emergence of the IBS from the Dirac cone. The spectral density has been calculated using the microscopic tight-binding model for an undoped $\left(E_{F}=0\right)$ semi-infinite region of graphene without disorder coupled to a superconductor. The distance from the interface is equivalent to the superconducting coherence length $x$. The white dashed line corresponds to the dispersion relation of (9). discontinuity between two regions described by the DBdG equation, taking a finite superconducting order parameter $\Delta$ and large doping $E_{F}^{S} \square \Delta$ on the superconducting side $(S)$ and zero order parameter and small doping $E_{F} \sim \Delta$ on the normal side $(N)$. For the analysis it is instructive to include an artificial intermediate normal region with $\Delta=0$ and $E_{F}^{I}=E_{F}^{S}$, whose width, $d$, can be taken to zero at the end of the calculation. This intermediate region allows to spatially separate normal reflection due to the Fermi energy mismatch from the Andreev reflection associated to the jump in $\Delta$. The DBdG equation reads

$$
\left(\begin{array}{cc}
H-E_{F} & \Delta \\
\Delta & E_{F}-H
\end{array}\right) \phi=E \phi
$$

with the excitation energy $E$ positive unless otherwise specified. The pair potential couples electron and holelike excitations from different valleys (opposite momentum), described by the same Hamiltonian

$$
H=-i \hbar v_{F}\left(\sigma_{x} \partial_{x}+\sigma_{y} \partial_{y}\right)
$$

We assume in (1) a s-wave pairing which leads to a constant gap $\Delta$ which is diagonal in sublattice space. Whenever the pair potential is assumed constant, the low energy spectrum is given by

$$
E=\sqrt{\Delta^{2}+\left(E_{F}-\hbar v_{F} \sqrt{k^{2}+q^{2}}\right)^{2}} .
$$

we define the component of the momentum perpendicular to the interface as

$$
\hbar v_{F} k_{e, h}^{S}=\sqrt{\left(E_{F}^{S} \pm \Omega\right)^{2}-\left(\hbar v_{F} q\right)^{2}},
$$

with $\Omega^{2}=E^{2}-D^{2}$ the conserved component of the momentum parallel to the interface. The basis of scattering states in the normal region $x<0$ with $\Delta=0$ is

$$
\begin{gathered}
\phi_{e+}=\left(1, \mathrm{e}^{i \alpha_{e}}, 0,0\right)^{T} \mathrm{e}^{i k_{e} x} \mathrm{e}^{i q y}, \\
\phi_{h+}=\left(0,0,1,-\mathrm{e}^{-i \alpha_{h}}\right)^{T} \mathrm{e}^{i k_{h} x} \mathrm{e}^{q y},
\end{gathered}
$$

for states moving towards the interface and

$$
\begin{aligned}
& \phi_{e^{-}}=\left(1,-\mathrm{e}^{-i \alpha_{e}}, 0,0\right)^{T} \mathrm{e}^{-i k_{e} x} \mathrm{e}^{i q y}, \\
& \phi_{h,-}=\left(0,0,1, \mathrm{e}^{-i \alpha_{h}}\right)^{T} \mathrm{e}^{-i k_{h} x} \mathrm{e}^{i q y},
\end{aligned}
$$

for states moving away from the interface. We define $\mathrm{e}^{i \alpha_{e, h}}=\hbar v_{F}\left(k_{e, h}+i q\right) /\left(E_{F} \pm E\right)$. Analogously, the basis of scattering states in the superconducting region $x>d$ with $\Delta \neq 0$ and $E_{F}^{S} \square \Delta$ reads

$$
\phi_{e+}^{S}=\left(u, u \mathrm{e}^{i \alpha_{e}^{S}}, v, v \mathrm{e}^{i \alpha_{e}^{S}}\right)^{T} \mathrm{e}^{i k_{e}^{S} x} \mathrm{e}^{i q y}
$$




$$
\phi_{h-}^{S}=\left(v,-v \mathrm{e}^{i \alpha_{h}^{S}}, u,-u \mathrm{e}^{i \alpha_{h}^{S}}\right)^{T} \mathrm{e}^{i k_{h}^{S} x} \mathrm{e}^{i q y}
$$

where $\mathrm{e}^{i \alpha_{e, h}^{S}}=\hbar v_{F}\left(k_{e, h}^{S}+i q\right) /\left(E_{F} \pm \Omega\right)$ and with

$$
u^{2}\left(v^{2}\right)=(E \pm \Omega) / 2 \Delta
$$

the BCS coherence factors, normalized so that $u^{2}+v^{2}=1$. Finally, for the intermediate region $0<x<d$ we use the normal state basis changing the doping level to $E_{F}^{I}$.

As shown in Figure 1 (case i), an incident electron from the normal side with energy $E$ and parallel momentum $\hbar q$ such that $\hbar v_{F}|q|>\left|E_{F}-E\right|$ is partially transmitted into the intermediate region and after a sequence of normal and Andreevreflections would be reflected as a hole. This process can either correspond to retro or specular Andreev reflection depending on whether $E<E_{F}$ or $E>E_{F}$ [8].

For $\hbar v_{F}|q|>\left|E_{F}-E\right|$ neither electron or holes can propagatewithin the graphene normal region. However, virtual processes like theone depicted in Figure 1 (case ii) would be present. These correspond to sequences of Andreev and normal reflections within the intermediate region. A bound state emergeswhen the total phase $\phi$ accumulated in such processes reach the resonancecondition $\phi=2 n \pi$.

It is quite straightforward to determine the dispersion relation for the IBS from the model represented in the top panel of Figure 1. The phase accumulated by a sequence of normal and Andreev reflections in the intermediate region can be obtained from the corresponding coefficients $r_{e}, r_{h}$ and $r_{A}$. From the matching of the solutions of the $\mathrm{DBdG}$ equations at the interface between the graphene and the intermediate regions one obtains

$$
r_{e, h}=\mathrm{e}^{i \alpha_{e, h}^{I}} \frac{\mathrm{e}^{-i \alpha_{e, h}^{I}}-\mathrm{e}^{-i \alpha_{e, h}}}{\mathrm{e}^{i \alpha_{e, h}^{I}}+\mathrm{e}^{-i \alpha_{e, h}}},
$$

where $\alpha_{e, h}^{(I)}=\arcsin \left[\hbar v_{F} q /\left(E \pm E_{F}^{(I)}\right)\right]$.

The condition $E_{F}^{I} \square \Delta, E, \hbar v_{F} q$ allows to take $\alpha_{e, h}^{I} \square 0$. On the other hand, in the region of evanescent electron and hole states for graphene $\left(\hbar v_{F}|q|>\left|E_{F}-E\right|\right)$, $r_{e, h}$ be- come a pure phase factor $\exp \left(i \varphi_{e, h}\right)$, with

$$
\begin{aligned}
& \varphi_{e, h}=-2 \operatorname{sgn}\left[q /\left(E \pm E_{F}\right)\right] \arctan \left[\exp \left(\lambda_{e, h}\right)\right] \\
& \text { and } \lambda_{e, h}=\operatorname{sgn}(q) \operatorname{argcosh}\left(\hbar v_{F} q /\left|E \pm E_{F}\right|\right) \text {. }
\end{aligned}
$$

For the Andreev reflection coefficient between regions $I$ and $S$ one has $r_{A}=\exp \left(i \varphi_{A}\right)$, where $\varphi_{A}=\arccos (E / \Delta)$, as it corresponds to the Andreev reflection at an ideal $N-S$ interface with $E_{F}^{S} \square \Delta$ [24]. In the limit $d \rightarrow 0$ the total phase accumulated is thus $\phi=2 \varphi_{A}+\varphi_{e}+\varphi_{h}$, from which one obtains the following dispersion relation

$$
\frac{E}{\Delta}= \pm \frac{\mathrm{e}^{\left(\lambda_{e}+\lambda_{h}\right) / 2}-\operatorname{sgn}\left(E^{2}-E_{F}^{2}\right) \mathrm{e}^{-\left(\lambda_{e}+\lambda_{h}\right) / 2}}{2 \sqrt{\cos \lambda_{e} \cos \lambda_{h}}} .
$$

This dispersion simplifies to

$$
E / \Delta= \pm \hbar v_{F} q / \sqrt{\left(\hbar v_{F} q\right)^{2}+\Delta^{2}}
$$

at the charge neutrality point (i.e. for $E_{F}=0$ ). In this case the IBS approaches zero energy for $q \rightarrow 0$ and tend asymptotically to the superconducting gap for large $q$. Notice also that the decay of the states into the graphene bulk region $(x<0$ in the top panel of Figure 1) is set by $\exp \left(x / \xi_{e, h}\right)$, where

$$
\xi_{e, h}=\hbar v_{F} /\left(\left|E \pm E_{F}\right| \sinh \lambda_{e, h}\right)
$$

for the electron and hole components respectively, which can be clearly much larger than the superconducting coherence length $\xi_{0}=\hbar v_{F} / \Delta$ when $E_{F} \square \Delta$. It is also interesting to notice that the IBSs survive when $E_{F}>\Delta$, i.e. in the regime corresponding to the usuSSal Andreev retroreflection, but with a much smaller spatial extension.

\section{Microscopic Model}

In order to describe the interface more microscopically, we analyze the electronic states of a graphene layer using the tight-binding approximation,

$$
H=-t_{g} \sum_{\langle i j\rangle} c_{i}^{\dagger} c_{j}+\sum_{i} V_{i} c_{i}^{\dagger} c_{i},
$$

where $t_{g}=2 \hbar v_{F} / 3 a_{0} \approx 2.6 \mathrm{eV}$ denotes the hopping element between nearest carbon atoms on the hexagonal lattice and $a_{0} \approx 0.142 \mathrm{~nm}$ is the smallest carbon-carbon distance. $V_{i}=V_{0}+\delta V_{i}$ is the potential applied to the lattice, where $V_{0}$ is a uniform on-site doping and $\delta V_{i}$ represents small fluctuations over the doping level (i.e. charge inhomogeneities as introduced below). The spin degree of freedom has been omitted due to degeneracy.

We assume that the graphene region is a strip with armchair edges along the $y$-direction as sketched in Figure 1. We model the strip by repeating a unit cell composed of four atoms $N$ times along the $x$ direction and $M$ times along the $y$ direction (see Figures 2(a) and (b)). As a consequence, the length of the graphene layer is $L=N \sqrt{3} a_{0}$. For describing the $W \square L$ limit we impose periodic boundary conditions in the $y$ direction and define $q \in\left[-\pi / d_{S C}, \pi / d_{S C}\right]$ as the corresponding wave vector, with $d_{S C}$ the vertical length of the supercell.

We connect the leftmost graphene armchair edge to a superconducting electrode and the rightmost to a normal lead. We maintain the graphene sublattice structure at the edges, thus representing the experimental situation where 
the electrodes are deposited on top of a graphene layer [16,25-28]. The presence of the superconducting correlations requires introducing the Nambu space, describing electron and hole propagation within the graphene layer. The self-energy on the graphene sites at the layer edge coupled to the normal lead is approximated by a $8 M \times 8 M$ matrix with elements $\Gamma_{i j, \alpha \beta, \mu \nu}^{R}=\delta_{i j} \delta_{\mu \nu}{ }^{R} \alpha \beta$, where $\alpha, \beta=1, \cdots, 4$ label the atomic sites within the unit cell, $i, j=1, \cdots, M$ label the unit cells in the superlattice and $\mu, v=e, h$ label the matrix elements in Nambu space. Following the geometry depicted in Figure 2, the elements of the self-energy matrix are explicitly defined as

$$
\gamma_{11, \mu \mu}^{R}=\gamma_{44, \mu \mu}^{R}=i \sqrt{3} / 2
$$

and $\quad \gamma_{14, e e}^{R}=\gamma_{41, e e}^{R}=-\gamma_{14, h h}^{R}=-\gamma_{41, h h}^{R}=-1 / 2 \quad$ (see more details in $[16,28])$.

Analogously, the self-energy describing the coupling to the superconducting electrode on the left armchair edge is described by a matrix with elements with $g_{B C S}=$

(a)

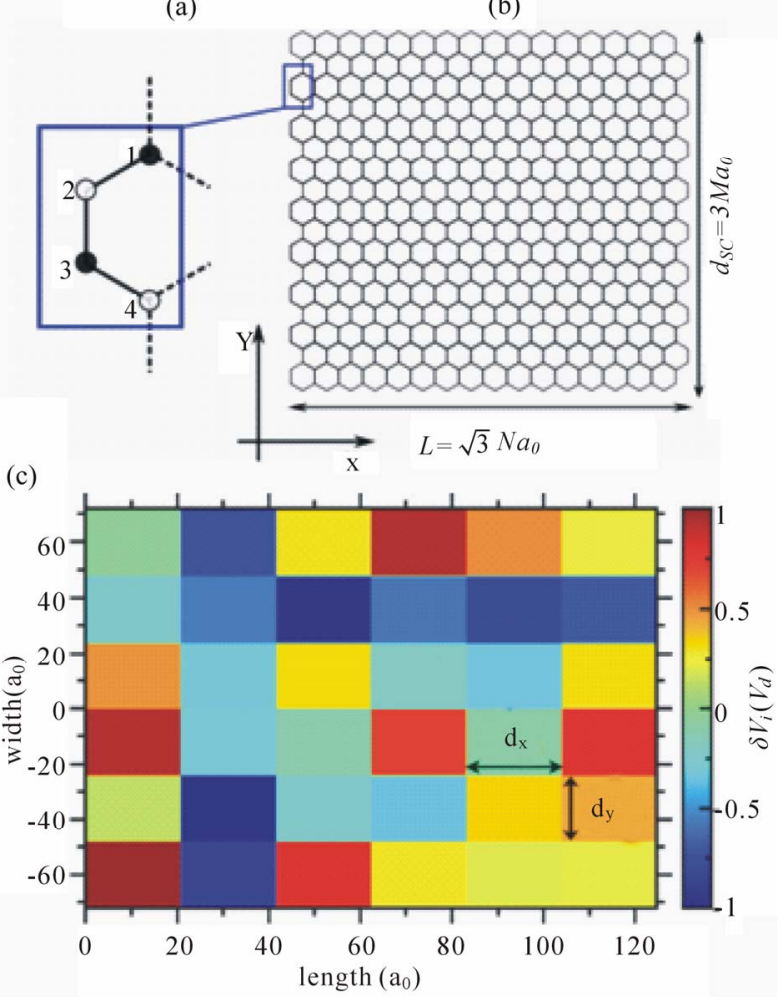

Figure 2. (a) Unit cell of the superlattice with four carbon atoms; (b) Graphene region showing the axis selection for $N$ $=17$ horizontal cells and $M=9$ vertical cells. The normal and superconducting electrodes would be coupled to the left and right armchair edges, respectively; (c) Example of a two dimensional superlattice potential with horizontal period $d_{x}$ and vertical period $d_{y}$ extended over a graphene region with $N=72$ and $M=48$ cells. A disordered superlattice potential takes random values in the range $\left[-V_{d}, V_{d}\right]$ at every square region given by $d_{x} \times d_{y}$.

$$
\begin{aligned}
& \Gamma_{22, \mu \mu}^{L}=\Gamma_{33, \mu \mu}^{L}=\sqrt{3} g_{B C S} / 2 \\
& \Gamma_{23, e e}^{L}=\Gamma_{33, e e}^{L}=-\Gamma_{23, h h}^{L}=-\Gamma_{32, h h}^{L}=-1 / 2 \\
& \Gamma_{22, e h}^{L}=\Gamma_{33, e h}^{L}=\Gamma_{22, h e}^{L}=\Gamma_{33, h e}^{L}=\sqrt{3} f_{B C S} / 2,
\end{aligned}
$$

$-E f_{B C S}=-E / \sqrt{\Delta^{2}-E^{2}}$ the BCS amplitudes and the superconducting gap.

The spectral properties of the system are calculated using the local retarded Green function $\hat{G}_{i i}^{r}(q, E)$, where $i=1, \cdots, N$ labels the horizontal sites of the layer. We can thus define the local density of states (LDOS) at the site $i$ as

$$
\rho_{i}(E)=\frac{d_{S C}}{(2 \pi)^{2}} \int_{-\pi / d_{S C}}^{\pi / d_{S C}} d_{q} \operatorname{Tr}\left[\operatorname{Im}\left\{\hat{G}_{i i}^{r}\right\}\right],
$$

which is normalized to one electron per site and spin. To remove the dependence on the width of the superlattice $d_{S C}$, it is convenient to normalize the LDOS with the density of a bulk graphene layer with zero doping at $E=\Delta, \quad \rho_{0}$, which for $\Delta \square \hbar v_{F} / d_{S C}$ is given by

$$
\Delta / 2 \pi\left(d_{S C} / \hbar v_{F}\right)^{2} \text {. }
$$

The results thus obtained do not depend on the ratio $\Delta / t_{g}$ used in our tight-binding calculations. In Figure 1(b) we show a map of the spectral density of states (the integrand of (11)) for an undoped $\left(E_{F}=0\right)$ semi-infinite region of graphene coupled to a superconductor. The energy-momentum map has been calculated at a distance to the interface comparable to the superconducting coherence length $\xi=\hbar v_{F} / \Delta$. The IBSs are located outside of the bulk band of graphene. The white dashed lines indicate the solution of (9).

\section{Model for Disorder Due to Charge Puddles}

The electron-hole inhomogeneity in graphene is modeled using a two-dimensional superlattice potential. As it is sketched in Figure 2(c), we divide the graphene layer into square supercells of horizontal length $\mathrm{d}_{x}=n \sqrt{3} a_{0}$ and vertical length $d_{y}=3 m a_{0}$, with $n \leq N$ and $m \leq M$. At each supercell, we define an electrostatic potential $\delta V_{i}$ which takes random values in the range $\left[-V_{d}, V_{d}\right]$, with $V_{d}$ the disorder strength. This random superlattice potential is added to the uniform electrostatic potential $V_{0}$. Consequently, $V_{0}$ stands for the doping level of the graphene layer and $\delta V_{i}$ represents the charge inhomogeneities characteristic of graphene (i.e., charge puddles $[29,30])$. Following [30], the best estimation for the maximum strength of the charge puddles is of $30 \mathrm{meV}$, over a region of typical size no greater than $30 \mathrm{~nm}$.

\section{Effect of Disorder}

When studying the low-energy physics of graphene close to the Dirac point, the electron-hole inhomogeneity in 
graphene (i.e., charge puddles $[29,30]$ ) is a type of charge disorder that has to be taken into account.

The results presented in Figure 1(b) correspond to a semi-infinite layer of pristine graphene connected to a superconductor. A more realistic model should include size effects such as a graphene layer with a finite length, and the possibility of electron-density inhomogeneities. Although the effect of having a finite length in the normal region can be treated within both the continuous and the TB models, the latter is more suitable to explore the effect of disorder.

The results for a graphene layer of horizontal length $L=\sqrt{3} \times 800 a_{0}$ with armchair edges coupled to a superconductor are presented in Figure 3. Figure 3(a) has been calculated with the strength of the disorder potential set to zero. The results for the LDOS (right panel) are similar to the undoped case presented in [16]: a clear peak at $E=\Delta$, which rapidly decays inside the normal region after a few times the superconducting coherence length $\xi$. The finite length of the graphene layer is manifested by the appearance of energy bands close to the gap edge. The spectral density of states is plotted in the left
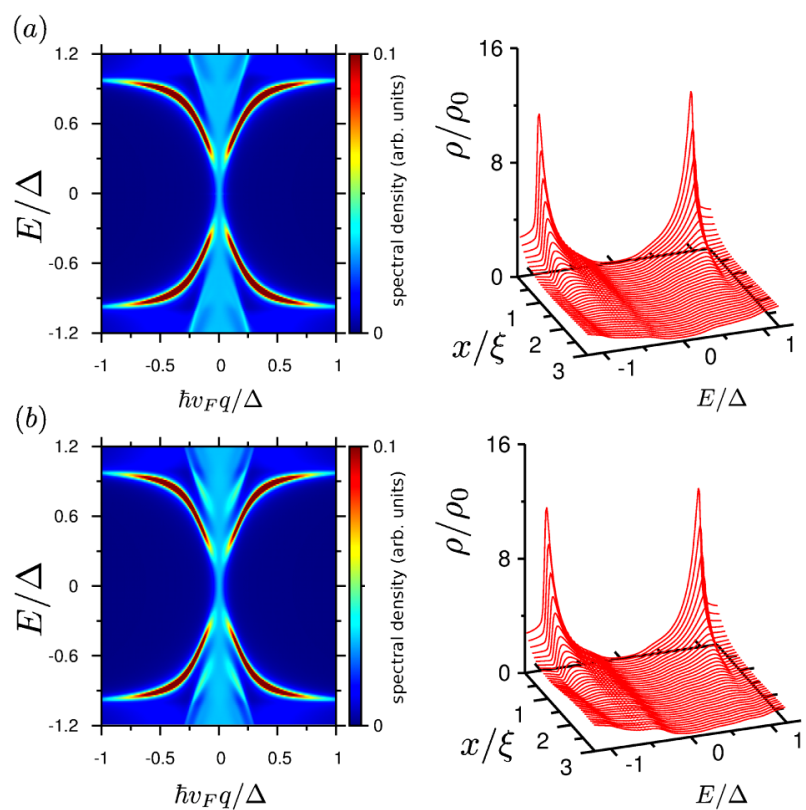

Figure 3. LDOS for a graphene layer of length $L=197 \mathrm{~nm}$ (800 armchair cells) coupled to a superconductor on an armchair edge calculated within the tight-binding model. For the simulation, the superconducting gap has been chosen to be $D=0.005 t_{g}$. (a) The results for the case without disorder. On the left, the spectral density of states, calculated at a distance from the interface comparable to the superconducting coherence length $x$, in a color map where dark blue is the absence of states. On the right, the LDOS as a function of the energy and the distance to the interface; (b) The same as before with the introduction of a random superlattice potential of strength $V_{d}=0.01 t_{g}$, with spatial periods $d_{x} \approx d_{y} \approx 10 \mathrm{~nm}$. panel, calculated at a distance $\xi$ from the interface. The IBS is clearly distinguishable outside of the Dirac cone (dark red in the color plot, the dark blue corresponds to the absence of spectral density). The finite armchair layer considered now presents a gap in the energy of the normal state. The Dirac cone is not uniform but is formed by discrete energy levels (light blue areas in the color plot) due to the finite size of the layer.

When the disorder is taken into account (Figure 3(b)), the peak at the edge of the gap remains unaltered and the decay is comparable to the non-disordered case (right panel). The weight of the states below the gap becomes more important in the spectral density, as it is shown in the left panel. The energy levels are deformed and acquire a bigger weight in the spectral density (light blue in the color plot).

For this strength of the disorder, the envelope of the LDOS is still comparable to the non-disordered case, but small fluctuations appear. The main effect of disorder can be seen as an effective doping, which does not alter deeply the LDOS. In Figure 4 we show the LDOS calculated at a distance from the interface $6 \xi$, where the IBS has completely decayed. The solid red line corresponds to the case where the disorder strength is set to zero. The LDOS presents a soft modulation and is symmetric with respect to the energy - as corresponds to an undoped case. The introduction of disorder breaks this electron-hole symmetry and clearly affects the modulation of the LDOS (blue dashed line).

The strength of the random superlattice potential used in these simulations is $V_{d}=0.01 t_{g} \approx 2.7 \mathrm{meV}$, which is comparable to the greatest estimation for the measured strength of the charge inhomogeneities in graphene [30]. The periodicity of the superlattice potential is $d_{x} \approx d_{y} \approx 10 \mathrm{~nm}$, which is slightly smaller than the typi-

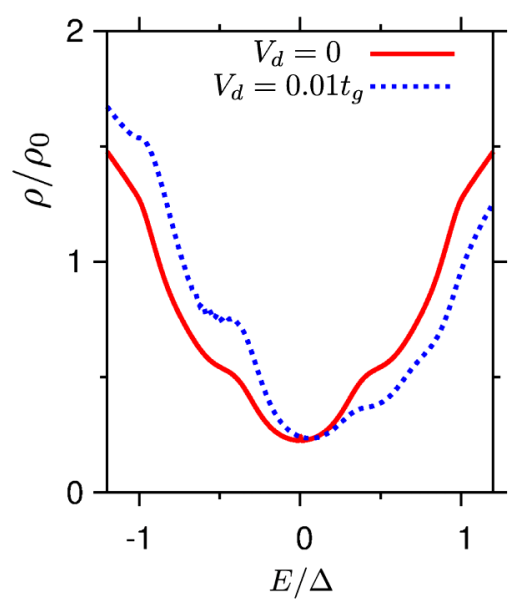

Figure 4. LDOS with the same parameters as in Figure 3 calculated at a distance $6 \times$ from the interface for the case without disorder (red solid line) and with disorder strength $V_{d}=0.01 t_{g}$ (blue dashed line). 
cal length of a charge puddle in graphene, with an average size of $30 \mathrm{~nm}$. In spite of this, the chosen values are close enough to assume that a bigger period for the superlattice potential would not affect considerably the LDOS profiles.

In conclusion, we have shown that interface bound states appear at isolated graphene-superconductor junctions. The presence of charge inhomogeneities in the normal region induces strong fluctuations in the LDOS profile and breaks the electron-hole symmetry of the LDOS. However, the IBS modifies more intensely the LDOS and thus this electron-hole symmetry cannot be appreciated at a distance from the interface comparable to $2-3 \xi$. For a longer distance, the IBS have decayed and the effect of the disorder is clearly shown in the LDOS. The formation of IBSs and their effect on the profile of the LDOS is robust against a disorder strength comparable to the measured strength of the charge puddles in graphene.

\section{Acknowledgements}

This work was supported by MICINN-Spain via grant FIS2008-04209 and EU project SE2ND (PB and ALY) and COLCIENCIAS, project 110152128235 (WJH).

\section{REFERENCES}

[1] S. Das Sarma, S. Adam, E. H. Hwang and E. Rossi, "Electronic Transport in Two-Dimensional Graphene," Reviews of Modern Physics, Vol. 83, No. 2, 2011, pp. 407-470. doi:10.1103/RevModPhys.83.407

[2] M. I. Katsnelson, K. S. Novoselov and A. K. Geim, "Chiral Tunnelling and the Klein Paradox in Graphene," Nature Physics, Vol. 2, 2006, p. 620. doi:10.1038/nphys 384

[3] C. W. J. Beenakker, "Colloquium: Andreev Reflection and Klein Tunneling in Graphene," Reviews of Modern Physics, Vol. 80, No. 4, 2008, pp. 1337-1354. doi:10.1103/RevModPhys.80.1337

[4] H. B. Heersche, P. Jarillo-Herrero, J. B. Oostinga, L. M. K. Vandersypen and A. F. Morpurgo, "Bipolar Supercurrent in Graphene," Nature (London), Vol. 446, 2007, p. 56. doi: $10.1038 /$ nature 05555

[5] F. Miao, S. Wijeratne, Y. Zhang, U. C. Coskun, W. Bao and C. N. Lau, "Phase-Coherent Transport in Graphene Quantum Billiards," Science, Vol. 317, No. 5844, 2007, pp. 1530-1533. doi:10.1126/science. 1144359

[6] A. Shailos, W. Nativel, A. Kasumov, C. Collet, M. Ferrier, S. Guèron, R. Deblock and H. Bouchiat, "Proximity Effect and Multiple Andreev Reflections in Few-Layer Graphene," EPL (Europhysics Letters), Vol. 79, No. 5, 2007, p. 57008. doi:10.1209/0295-5075/79/57008

[7] X. Du, I. Skachko and E. Y. Andrei, "Josephson Current and Multiple Andreev Reflections in Graphene SNS Junctions," Physical Review B, Vol. 77, No. 18, 2008, Article

\section{ID: 184507. doi:10.1103/PhysRevB.77.184507}

[8] C. W. J. Beenakker, "Specular Andreev Reflection in Graphene," Physical Review Letters, Vol. 97, No. 6, 2006, Article ID: 067007. doi:10.1103/PhysRevLett.97.067007

[9] Y. Li and N. Mason, "Tunneling Spectroscopy of Graphene Using Planar Pb Probes," 2012. arXiv:1210.4987 [cond-mat.meshall].

[10] C. W. J. Beenakker, R. A. Sepkhanov, A. R. Akhmerov and J. Tworzydlo, "Quantum Goos-Hänchen Effect in Graphene," Physical Review Letters, Vol. 102, No. 14, 2009, Article ID: 146804. doi:10.1103/PhysRevLett.102.146804

[11] S. Bhattacharjee and K. Sengupta, "Tunneling Conductance of Graphene NIS Junctions," Physical Review Letters, Vol. 97, No. 21, 2006, Article ID: 217001. doi:10.1103/PhysRevLett.97.217001

[12] A. R. Akhmerov and C. W. J. Beenakker, "Pseudodiffusive Conduction at the Dirac Point of a Normal-SuperconDuctor Junction in Graphene," Physical Review B, Vol. 75, No. 4, 2007, Article ID: 045426. doi:10.1103/PhysRevB.75.045426

[13] G. Tkachov, "Fine Structure of the Local Pseudogap and Fano Effect for Superconducting Electrons near a Zigzag Graphene Edge," Physical Review B, Vol. 76, No. 23, 2007, Article ID: 235409. doi:10.1103/PhysRevB.76.235409

[14] J. Linder and A. Sudbø, "Dirac Fermions and Conductance Oscillations in \$s\$- and \$d \$-Wave SuperconductorGraphene Junctions," Physical Review Letters, Vol. 99, No. 14, 2007, Article ID: 147001. doi:10.1103/PhysRevLett.99.147001

[15] J. Linder and A. Sudbø, "Tunneling Conductance in \$s\$and \$d\$-Wave Superconductor-Graphene Junctions: Extended Blonder-Tinkham-Klapwijk Formalism," Physical Review B, Vol. 77, No. 14, 2008, Article ID: 064507. doi:10.1103/PhysRevB.77.064507

[16] P. Burset, A. L. Yeyati and A. Martín-Rodero, "Microscopic Theory of the Proximity Effect in SuperconductorGraphene Nanostructures," Physical Review B, Vol. 77, No. 20, 2008, Article ID: 205425. doi:10.1103/PhysRevB.77.205425

[17] J. Cserti, I. Hagymási and A. Kormányos, "Graphene Andreev Billiards," Physical Review B, Vol. 80, No. 7, 2009, Article ID: 073404. doi:10.1103/PhysRevB.80.073404

[18] D. Rainis, F. Taddei, F. Dolcini, M. Polini and R. Fazio, "Andreev Reflection in Graphene Nanoribbons," Physical Review B, Vol. 79, No. 11, 2009, Article ID: 115131. doi:10.1103/PhysRevB.79.115131

[19] Q.-F. Sun and X. C. Xie, "Quantum Transport through a Graphene Nanoribbon-Superconductor Junction," Journal of Physics: Condensed Matter Vol. 21, No. 34, 2009, Article ID: 344204. doi:10.1088/0953-8984/21/34/344204

[20] S.-G. Cheng, H. Zhang and Q.-F. Sun, "Effect of Electron-Hole Inhomogeneity on Specular Andreev Reflection and Andreev Retroreflection in a Graphene-Superconductor Hybrid System," Physical Review B, Vol. 83, No. 23, 2011, Article ID: 235403. 


\section{doi:10.1103/PhysRevB.83.235403}

[21] M. Titov, A. Ossipov and C. W. J. Beenakker, "Excitation Gap of a Graphene Channel with Superconducting Boundaries," Physical Review B, Vol. 75, No. 4, 2007, Article ID: 045417. doi:10.1103/PhysRevB.75.045417

[22] D. L. Bergman and K. Le Hur, "Near-Zero Modes in Condensate Phases of the Dirac Theory on the Honeycomb Lattice," Physical Review B, Vol. 79, No. 18, 2009, Article ID: 184520. doi:10.1103/PhysRevB.79.184520

[23] P. Burset, W. Herrera and A. L. Yeyati, "Proximity-Induced Interface Bound States in Superconductor-Graphene Junctions," Physical Review B, Vol. 80, No. 4, 2009, Article ID: 041402. doi:10.1103/PhysRevB.80.041402

[24] G. E. Blonder, M. Tinkham and T. M. Klapwijk, "Transition from Metallic to Tunneling Regimes in Superconducting Microconstrictions: Excess Current, Charge Imbalance, and Supercurrent Conversion," Physical Review $B$, Vol. 25, No. 7, 1982, pp. 4515-4532. doi:10.1103/PhysRevB.25.4515

[25] H. Schomerus, "Effective Contact Model for Transport through Weakly-Doped Graphene," Physical Review B, Vol. 76, No. 4, 2007, Article ID: 045433.

\section{doi:10.1103/PhysRevB.76.045433}

[26] Y. M. Blanter and I. Martin, "Transport through NormalMetal Graphene Contacts," Physical Review B, Vol. 76, No. 15, 2007, Article ID: 155433. doi:10.1103/PhysRevB.76.155433

[27] L. Brey and H. A. Fertig, "Magnetoresistance of Graphene-Based Spin Valves," Physical Review B, Vol. 76, No. 20, 2007, Article ID: 205435. doi:10.1103/PhysRevB.76.205435

[28] P. Burset, A. L. Yeyati, L. Brey and H. A. Fertig, "Transport in Superlattices on Single-Layer Graphene," Physical Review B, Vol. 83, No. 19, 2011, Article ID: 195434. doi:10.1103/PhysRevB.83.195434

[29] J. Martin, N. Akerman, G. Ulbricht, T. Lohmann, J. H. Smet, K. Von Klitzing and A. Yacoby, "Observation of Electron-Hole Puddles in Graphene Using a Scanning Single-Electron Transistor," Nature Physics, Vol. 4, No. 2, 2008, pp. 144-148. doi:10.1038/nphys781

[30] Y. Zhang, V. W. Brar, C. Girit, A. Zettl and M. F. Crommie, "Origin of Spatial Charge Inhomogeneity in Graphene," Nature Physics, Vol. 5, No. 10, 2009, pp. 722726. doi:10.1038/nphys 1365 